\title{
Desinfección de Tubérculos-Semilla de Papa infectados con Nacobbus aberrans Utilizando Productos Químicos en Aspersión a Ultra Bajo Volumen $^{1}$
}

Javier Franco, Gladys Main ${ }^{2}$

\begin{abstract}
Resumen
Tubérculos de papa de la variedad Waych'a (Solanum tuberosum ssp. andigena) provenientes de parcelas con $80 \%$ de infección radical por Nacobbus aberrans fueron tratados mediante una máquina de aplicación a Ultra Bajo Volumen (UBV), con tres diferentes dosis de los productos comerciales, Cierto 90 EC (Fostiazate 90\%: 18, 12 y 6 cc/l), Vertimec 018 EC (Abamectin 18\%: 37.5, 25.0 y $12.5 \mathrm{cc} / \mathrm{l}$ ) y Nemacur 40 EC (Fenamiphos 40\%: 37.5, 25.0 y $12.5 \mathrm{cc} / \mathrm{l}$ ) y en inmersión a las dosis más bajas. Los tubérculos tratados fueron evaluados en bioensayos de bolsa cerrada y en macetas a temperatura ambiente y a $22^{\circ} \mathrm{C}$. Los resultados mostraron que todos los tratamientos de inmersión fueron efectivos para eliminar la presencia de $N$. aberrans en los tubérculos. El tratamiento a UBV con Nemacur no eliminó el nematodo de los tubérculos, como sí ocurrió con los tratamientos con Vertimec y Fostiazate. Así mismo, se comprobó que en las raíces de las plantas sin tratar hubo formación de nódulos radicales. No se observaron efectos fitotóxicos en el desarrollo de las plantas. Estos resultados muestran que es posible la eliminación de $N$. aberrans en tubérculos-semilla de papa por su tratamiento a UBV con los productos Abamectin (Vertimec $018 \mathrm{EC}$ ) y Fostiazate (Cierto $90 \mathrm{EC}$ ).
\end{abstract}

Palabras claves adicionales: Nematodos de papa, control químico, Nematicidas.

1 Consultaría efectuada a la Empresa de Semilla de Papa-SEPA, Cochabamba, Bolivia.

2 Fundación PROINPA (Producción e Investigación de Productos Andinos), Casilla 4285, Cochabamba, Bolivia. 


\title{
Disinfection of Potato Seed Tubers Infected with Nacobbus aberrans Using Chemical Compounds with ULV Sprays
}

\begin{abstract}
Summary
Tubers collected from heavily Nacobbus aberrans infected potato plants ( $80 \%$ root nodulation) of cv. Waycha (Solanum tuberosum ssp. andigena) were treated with different chemical compounds by using a ULV machine in Cochabamba, Bolivia. The evaluated compounds were applied as ULV at three different dosages of the commercial products: Cierto 90 EC (Fostiazate 90\%: 18, 12, $6 \mathrm{cc} / \mathrm{l}$ ), Vertimec 018 EC (Abamectin 18\%: 37.5, 25.0, 12.5 cc/l) y Nemacur 40 EC (Fenamiphos 40\%: 37.5, 25.0, $12.5 \mathrm{cc} / \mathrm{l}$ ) and as tuber inmersion at the lower dosages. Treated and untreated tubers were evaluated as bioassays in plastic bags and clay pots containing sterile soil at greenhouse temperature and $22^{\circ} \mathrm{C}$. Results showed that tuber inmersion in all cases was very effective in eliminating $N$. aberrans in infected tubers. On the contrary, with ULV treatments Nemacur was not so effective as tubers treated with Vertimec and Fostiazate. $N$. aberrans nodules in roots of control plants confirmed the efficiency of tuber treatments. No phytotoxic effects were observed during sprouting or plant development. These results show that $N$. aberrans elimination from potato seed-tubers can be effectively obtained by their ULV treatment with Abamectin (Vertimec 018 EC) and Fostiazate (Cierto $90 \mathrm{EC}$ ).
\end{abstract}

Additional Index words:

Potato nematodes, chemical control, nematicides.

\section{Introducción}

La ocurrencia de tubérculos infectados con $N$. aberrans es común en la región andina de Bolivia y Perú y en el nor-oeste de la Argentina $(1,4)$. Estos nematodos penetran 1-2 mm por debajo de la piel del tubérculo y permanecen en estado de latencia por un periodo de cinco a seis meses, lo cual les permite sobrevivir y diseminarse efectivamente con los tubérculos empleados como semillas (2).

N. aberrans causa pérdidas de aproximadamente US\$53 millones al año en las regiones alto-andinas destinadas al cultivo de la papa en Bolivia. Las mermas en el rendimiento, alcanzan hasta el $61.5 \%$ además de ocasionar 
gastos adicionales por el control a efectuarse y por el diagnóstico para verificar su presencia (4); además, ocasiona la descalificación de los campos destinados a la producción formal de semilla (6). Las Normas Vigentes de Certificación de tubérculos-semillas de papa en Bolivia, establecen una tolerancia de cero a $N$. aberrans, en las categorías Básica, Registrada y Certificada, y la categoría Fiscalizada se establece a partir de la descalificación de las categorías anteriores por presencia de $N$. aberrans (9).

Por otro lado, de no evitarse la diseminación de $N$. aberrans por medio de tubérculos infectados a zonas aun libres, la producción de papa continuará siendo afectada severamente, como ocurre con los tubérculos provenientes del sistema informal de "semilla", que se comercializan a través de las ferias locales y regionales, que constituyen las principales fuentes de diseminación de este nematodo y otros patógenos $(14,17)$.

Se ha determinado que existe una relación directa entre las densidades de población de $N$. aberrans en el suelo, la nodulación radical en planta y la infección de los tubérculos, aunque estos últimos no son infectados total y uniformemente. Los tubérculos procedentes de campos con una incidencia de plantas noduladas menor al $10 \%$ en relación al número total de plantas muestreadas y con una severidad de nodulación radical de las mismas plantas menor al 5\% de acuerdo al volumen total del sistema radical de cada una de ellas, presentan una nula o incipiente incidencia de tubérculos infectados $(5,15)$.

Por otro lado, estudios efectuados en campo para el control de $N$. aberrans con productos químicos aplicados a la siembra demostraron ser efectivos en reducir las pérdidas en el rendimiento y la población de nematodos en los tubérculos, pero no eliminaron su presencia en los tubérculos-semillas $(12,18)$.

Con relación al tratamiento de tubérculos-semillas infectados con productos químicos, se han efectuados diversos estudios. Así, Costilla et al. (3) indica que la inmersión de los tubérculos en formulaciones líquidas de los nematicidas Nemacur (Fenamiphos 40\%) y Mocap (Ethoprop 75\%) a 2000 ppm del producto comercial por 10 minutos, pueden ser usados exitosamente para evitar la diseminación de $N$. aberrans a campos no infestados.

De esta forma se seleccionaron aquellos productos que por pruebas sucesivas y diferentes periodos de inmersión confirmaron su efectividad en la eliminación de $N$. aberrans en tubérculos, aun cuando el proceso de inmersión seguía constituyendo una limitación, tanto por el empleo de productos químicos de alta toxicidad, como por el proceso mismo de inmersión de grandes volúmenes de tubérculos.

Resultados de evaluaciones efectuadas a los 80 días después de la siembra de tubérculos tratados con diversos productos químicos, mostraron que la inmersión en Carbodan 48FW a las dosis de 12.5 y 25 cc/l y Mocap 
91.5 a $1.5 \mathrm{cc} / \mathrm{l}$ durante 10 minutos, fueron los que mejor controlaron la formación de nódulos radicales (10). Así mismo, se confirmó la efectividad del Nemacur 40EC por la inmersión de tubérculos provenientes de parcelas con $10 \%$ de incidencia y $5 \%$ de severidad de nodulación radical por N. aberrans (5).

Posteriormente, tratamientos de inmersión realizados con productos de menor toxicidad como Azatin (azadirachtin), Cipersen (cipermetrina), Promet (furatecarb) y Vertimec (abamectin), mostraron una eficiencia similar al Nemacur 40EC para controlar N. aberrans en tubérculos (8).

El empleo del calor seco y/o húmedo por un determinado periodo de tiempo, ha proporcionado resultados contradictorios en cuanto a la eliminación de $N$. aberrans de tubérculos infectados. Tal como se obtuvieron resultados favorables sobre la presencia del nematodo, no fue así sobre la calidad de los tubérculos-semillas, en el que el brotamiento de los mismos en algunos casos fue severamente afectado (13). Por otro lado, se observó una mayor nodulación por $N$. aberrans en plantas de tubérculos sometidos a termoterapia que con los testigos, por lo que se presume que la temperatura y tiempo empleados no fue letal para los nematodos, sino por el contrario activaron al nematodo y favorecieron una mayor formación de nódulos radicales (7).

Sin embargo, aun cuando se conoce la efectividad de la inmersión de tubérculos en diversos productos químicos, en la actualidad es importante el identificar productos menos tóxicos que puedan ser empleados efectivamente en el tratamiento de tubérculos-semillas infectados por $N$. aberrans sin poner en riesgo la salud humana, la biodiversidad y el medio ambiente.

En el presente estudio, se evaluó la efectividad de la aplicación mecánica de UBV de los productos Vertimec y Cierto en el tratamiento de tubérculos provenientes de campos infestados por $N$. aberrans como una alternativa eficiente y segura, que pueda ser empleada por los productores de tubérculos-semillas, sin el peligro de diseminar esta plaga. Además, se estudió por cinco meses consecutivos la declinación natural de N. aberrans en tubérculos infectados procedentes del mismo lote de los empleados para su tratamiento químico.

\section{Materiales y Métodos}

Tubérculos de papa (Solanum tuberosum ssp. andigená) del cv. W'aycha provenientes de parcelas con $80 \%$ de infección radical producida por $N$. aberrans, fueron tratados mediante una máquina de aplicación a Ultra Bajo Volumen (UBV) y la inmersión en los productos comerciales en base Fostiazate 90\%, Abamectin 18\% y Fenamiphos $40 \%$ a diferentes dosis, tal como se indica en detalle en la Tabla 1. 


\section{Tabla 1. Productos y dosis evaluadas para el tratamiento de tubérculos-semillas de papa infectados por $N$. aberrans.}

\begin{tabular}{|c|c|c|c|c|}
\hline Tratamiento & $\begin{array}{l}\text { Ingrediente activo } \\
\text { (concentración) }\end{array}$ & Nivel & $\begin{array}{l}\text { Cantidad } \\
\text { (cc/litro) }\end{array}$ & $\begin{array}{l}\text { Forma de } \\
\text { aplicación }\end{array}$ \\
\hline 1. Cierto EC & Fostiazate90\% & Alta & 18.0 & $(\text { UBV })^{1}$ \\
\hline 2. Cierto EC & Fostiazate90\% & Media & 12.0 & (UBV) \\
\hline 3. Cierto EC & Fostiazate $90 \%$ & Baja & 6.0 & (UBV) \\
\hline 4. Cierto EC & Fostiazate $90 \%$ & Baja & 6.0 & $(\text { Inmersión) })^{2}$ \\
\hline 5. Vertimec EC & Abamectin $18 \%$ & Alta & 37.5 & (UBV) \\
\hline 6. Vertimec EC & Abamectin $18 \%$ & Media & 25.0 & (UBV) \\
\hline 7. Vertimec EC & Abamectin $18 \%$ & Baja & 12.5 & (UBV) \\
\hline 8. Vertimec EC & Abamectin $18 \%$ & Baja & 12.5 & (Inmersión) \\
\hline 9. Nemacur EC & Fenamiphos 40\% & Alta & 37.5 & (UBV) \\
\hline 10. Nemacur EC & Fenamiphos $40 \%$ & Media & 25.0 & (UBV) \\
\hline 11. Nemacur EC & Fenamiphos $40 \%$ & Baja & 12.5 & (UBV) \\
\hline 12. Nemacur EC & Fenamiphos $40 \%$ & Baja & 12.5 & (inmersión) \\
\hline 13. Testigo agua & 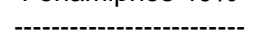 & --- & --- & (UBV) \\
\hline 14. Testigo agua & ---------------------------- & ----- & ----- & (Inmersión) \\
\hline 15. Testigo seco & 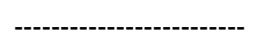 & -.--- & -.--- & (Oscuridad) \\
\hline 16. Testigo seco ${ }^{3}$ & ----------------------------- & ----- & ----- & (Luz difusa) \\
\hline 17. Testigo seco ${ }^{3}$ & |------------------------- & ---- & ---- & (Verdeo inicio) \\
\hline
\end{tabular}

1: Requirió de 200 ce de solución preparada para cada tratamiento; 2: Inmersión por 10 minutos en 3 I de solución; 3: Considerados únicamente en bioensayos con bolsas a $22^{\circ} \mathrm{C}$ y macetas.

El producto Vertimec 018 EC es un insecticida-acaricida biológico, de acción translaminar y de ligera toxicidad oral y dermal, con mínimo efecto sobre enemigos naturales. En contraste, los productos nematicidas Cierto EC y Nemacur EC son órgano-fosforados de acción sistémica y con alta toxicidad oral y dermal, por lo que su uso requiere de los cuidados y precauciones que se recomiendan para este tipo de productos.

La máquina empleada para generar el UBV es de marca MAFEX 870-1 de fabricación alemana (MANTIS ULV), con un sistema de bombeo y aplicación a UBV que se calibró para un gasto de aplicación de los tratamientos a emplearse de $40 \mathrm{cc} / \mathrm{min}$, volumen que permitió producir una neblina en el túnel de la máquina para tratar 3 toneladas de tubérculossemilla de papa por hora. El paso de los tubérculos a través del túnel se efectuó por la adaptación de una mesa transportadora marca NICAMESA de $1.60 \mathrm{~m}$ de largo y $0.80 \mathrm{~m}$ de ancho, que posee 15 rodillos forrados en goma de $8.12 \mathrm{~cm}$ y con un espacio entre rodillos de $1.16 \mathrm{~cm}$ que giran a una velocidad de $50 \mathrm{rpm}$.

Posteriormente, los tubérculos tratados fueron almacenados en el ambiente oscuro de un silo y una vez iniciado el brotamiento, estos fueron evaluados individualmente por bioensayos en bolsas de plástico y macetas. Los tubérculos brotados fueron sembrados en bolsas de plástico transparente conteniendo $400 \mathrm{cc}$ de suelo estéril y con una humedad de capacidad de 
campo. Seguidamente se sellaron las bolsas y se conservaron bajo oscuridad, tanto en un silo a temperatura de medio ambiente como en un cuarto temperado a $22^{\circ} \mathrm{C}$. Estos bioensayos fueron evaluados por la presencia de nódulos radicales a los 49 días después de la siembra. En forma paralela y para comparar con los bioensayos en bolsa, bajo condiciones de invernadero se sembraron tubérculos tratados en macetas de 1000 ce conteniendo también suelo estéril, los mismos que fueron evaluados en forma similar al caso de las bolsas, pero a los 125 días después de la siembra. En todos los casos, los bioensayos fueron evaluados de acuerdo a la escala de grado de ataque que se presenta en la Tabla 2.

Tabla 2. Escala de calificación del grado de ataque y del nivel de severidad por $\mathrm{N}$. aberrans en base al número de nódulos presentes en el sistema radical de una planta de papa.

\begin{tabular}{c|cc}
\hline Grado de ataque & Número de nódulos & Nivel de severidad \\
\hline 0 & 0 & Libre \\
1 & $1-10$ & Incipiente \\
2 & $11-30$ & Moderado \\
3 & $31-75$ & Intenso \\
4 & $>75$ & Muy intenso \\
\hline
\end{tabular}

Fuente: Franco (4).

Para determinar la población inicial y el grado de declinación natural de $N$. aberrans en tubérculos no tratados a causa del almacenamiento se procesaron mensualmente y en forma individual 20 tubérculos en 5 oportunidades (cinco meses). Cada tubérculo se procesó mediante el método del macerado que consiste en extraer la cáscara de cada tubérculo previamente lavado, para luego licuar la cáscara durante 30 segundos con 400 cc de agua y unas gotas de detergente para romper la tensión superficial. Luego esta solución se pasó a través de una batería de tamices de 100 y 400 mesh: el primero para recolectar la parte gruesa y el segundo para recolectar a los nematodos. Este último material se colocó en tubos de centrífuga para luego llenarlos con una solución azucarada (50\%) y centrifugarlos a $3000 \mathrm{rpm}$ durante 3 minutos, para luego pasar la suspensión por tamices de 400 mesh y los nematodos finalmente se recolectaron en pequeños frascos con la menor cantidad de agua posible, para su posterior observación al microscopio estereoscópico (11).

Las variables cuantitativas de los bioensayos (número de nódulos radicales por planta) fueron procesadas como experimento factorial en un diseño completamente al azar y para realizar comparaciones entre los tratamientos se aplicaron contrastes ortogonales (16). 


\section{Resultados y Discusión}

\section{Población inicial y declinación natural de nematodos en tubérculos en almacenamiento}

Los resultados obtenidos con los tubérculos no tratados, que fueron evaluados mensualmente por un periodo de almacenamiento de cinco meses, muestran en primer lugar (Figura 1) que al inicio del estudio en el mes de mayo (antes del tratamiento químico), el 100\% de los tubérculos $(n=20)$ se encontraban infectados por $N$. aberrans. El $60 \%$ de estos tubérculos presentaban un número relativamente moderado de nematodos (1-50 especímenes/tubérculo); el 20\% con una infección alta (51-100) y el otro $20 \%$ con una infección muy alta (entre 100 y 250 especímenes por tubérculo). Así mismo se ha observado que a medida que transcurre el periodo de almacenamiento, ocurre una declinación o muerte natural de la población de nematodos en los tubérculos. Se observó que a partir del mes de junio en cierto porcentaje de tubérculos ya no hay nematodos; finalmente en septiembre se tiene un $30 \%$ de tubérculos libres de nematodos y un $70 \%$ de tubérculos con una infección moderada, pero capaz de provocar o dar origen a plantas con nodulación radical, tal como se confirma por los resultados obtenidos con los tubérculos testigos en la prueba con productos químicos (Tabla 3).

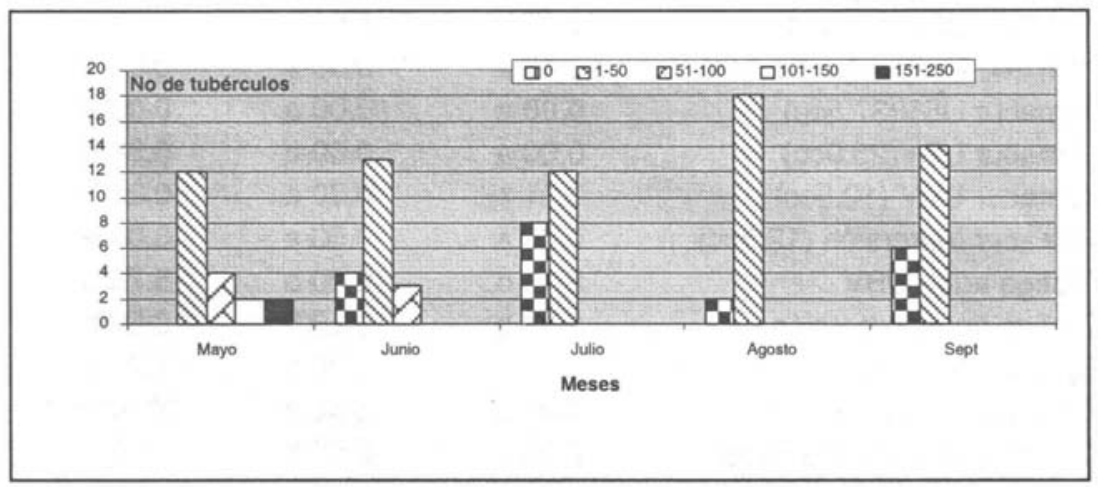

Figura 1. Declinación natural en la incidencia y severidad de N. aberrans en tubérculos semillas de papa $(n=20)$ almacenados en oscuridad durante 5 meses.

\section{Efecto de los productos químicos sobre la población de $N$. aberrans en tubérculos}

Las comparaciones ortogonales (Tabla 4) realizadas con los resultados de nodulación radical obtenidos en las pruebas de bioensayo en bolsas de plástico o en macetas, muestran que en todos los casos los testigos no 
tratados fueron significativamente diferentes a los productos químicos empleados, tanto en la inmersión de los tubérculos como con la aspersión UBV de los mismos. Por estos resultados se comprobó, además, que las tres pruebas de bioensayo empleadas fueron favorables para la formación de nódulos radicales en las raíces de las plantas sin tratar, aun cuando sobresalió el bioensayo en macetas. Este último permitió la formación de nódulos radicales con mayor eficiencia.

Tabla 3. Número promedio de nódulos radicales en bioensayos (bolsas de plástico y macetas) por efecto de los diversos tratamientos (Duncan 0.05).

\begin{tabular}{cccc}
\hline & \multicolumn{3}{c}{ Bioensayos } \\
\cline { 2 - 4 } Tratamientos & $\begin{array}{c}\text { Bolsas } \\
(\text { Temp. amb. })^{1}\end{array}$ & $\begin{array}{c}\text { Bolsas } \\
\left(22^{\circ} \mathrm{C}\right)^{2}\end{array}$ & $\begin{array}{c}\text { Macetas } \\
(\text { Temp. amb. })^{3}\end{array}$ \\
\hline Fostiazate UBV(18cc) & $0.00 \mathrm{a}^{4}$ & $0.00 \mathrm{a}$ & $0.00 \mathrm{a}$ \\
Fostiazate UBV(12cc) & $0.00 \mathrm{a}$ & $0.00 \mathrm{a}$ & $0.00 \mathrm{a}$ \\
Fostiazate UBV(6cc) & $0.00 \mathrm{a}$ & $0.00 \mathrm{a}$ & $0.00 \mathrm{a}$ \\
Fostiazate Inmersión (6cc) & $0.00 \mathrm{a}$ & $0.00 \mathrm{a}$ & $0.00 \mathrm{a}$ \\
Vertimec UBV(37.5cc) & $0.00 \mathrm{a}$ & $0.00 \mathrm{a}$ & $0.00 \mathrm{a}$ \\
Vertimec UBV(25.0cc) & $0.00 \mathrm{a}$ & $0.00 \mathrm{a}$ & $0.00 \mathrm{a}$ \\
Vertimec UBV(1 2. 5cc) & $0.00 \mathrm{a}$ & $0.00 \mathrm{a}$ & $0.00 \mathrm{a}$ \\
Vertimec Inmersión (12.5cc) & $0.00 \mathrm{a}$ & $0.00 \mathrm{a}$ & $0.00 \mathrm{a}$ \\
Nemacur UBV(37.5cc) & $0.00 \mathrm{a}$ & $0.00 \mathrm{a}$ & $0.00 \mathrm{a}$ \\
Nemacur UBV(25.0cc) & $0.00 \mathrm{a}$ & $0.00 \mathrm{a}$ & $0.33 \mathrm{~b}$ \\
Nemacur UBV (12.5cc) & $0.11 \mathrm{a}$ & $0.00 \mathrm{a}$ & $0.25 \mathrm{~b}$ \\
Nemacur Inmersión (12.5cc) & $0.00 \mathrm{a}$ & $0.00 \mathrm{a}$ & $0.00 \mathrm{a}$ \\
Testigo agua UBV & $2.11 \mathrm{~b}$ & $0.00 \mathrm{a}$ & $5.75 \mathrm{~b}$ \\
Testigo agua inmersión & $2.33 \mathrm{~b}$ & $0.50 \mathrm{~b}$ & $2.50 \mathrm{~b}$ \\
Testigo seco (oscuridad) & $2.60 \mathrm{~b}$ & $1.00 \mathrm{~b}$ & $1.25 \mathrm{~b}$ \\
Testigo seco (luz difusa) & $0.00 \mathrm{a}$ & $6.40 \mathrm{~b}$ & $10.40 \mathrm{~b}$ \\
Testigo seco (verdeo inicial.) & $0.00 \mathrm{a}$ & $5.60 \mathrm{~b}$ & $4.8 \mathrm{~b}$ \\
\hline
\end{tabular}

1= 10 repeticiones; $2=5$ repeticiones; $3=5$ repeticiones; $4=$ Tratamientos con letras diferentes son estadísticamente significativos (Duncan 0.05). 
Tabla 4. Comparaciones (contrastes ortogonales) de los testigos contra los diversos tratamientos.

$$
\text { (p } \left.0.05>\text { chi }^{2}\right)
$$

\begin{tabular}{lccc}
\hline \multicolumn{1}{c}{ Contrastes } & \multicolumn{3}{c}{ Niveles de significación } \\
\hline & $\begin{array}{c}\text { Bolsas } \\
(\text { Temp. amb.) }\end{array}$ & $\begin{array}{c}\text { Bolsas } \\
\left(22^{\circ} \mathrm{C}\right)\end{array}$ & $\begin{array}{c}\text { Macetas } \\
(\text { Temp. amb. })\end{array}$ \\
\hline Testigos vs. Todos & $0.0001^{\star}$ & $0.0001^{*}$ & $0.0104^{\star}$ \\
Testigos vs. Vertimec & $0.0001^{*}$ & $0.0003^{*}$ & $0.0124^{\star}$ \\
Testigos vs. Fostiazate & $0.0001^{*}$ & $0.0003^{*}$ & $0.0037^{*}$ \\
Testigos vs. Nemacur & $0.0001^{*}$ & $0.0001^{*}$ & 0.3298 \\
\hline
\end{tabular}

Los análisis estadísticos de comparación de medias por la prueba de Duncan (Tabla 4), confirman que la inmersión por 10 minutos de tubérculos infectados con $N$. aberrans en las dosis estudiadas de los productos Nemacur 40EC, Fostiazate 90EC y Vertimec 018EC fueron efectivos en la eliminación del nematodo de los tubérculos, ya que no se observó la formación de nódulos radicales en las plantas de papa originadas de los tubérculos tratados. Similarmente, la aspersión a ultra bajo volumen con las dosis estudiadas de los productos Fostiazate 90EC y Vertimec 018EC también fueron efectivas. Sin embargo, es importante indicar de ciertas divergencias que se observaron en los resultados obtenidos en los bioensayos con respecto al producto Nemacur aplicado a UBV, que pueden atribuirse al efecto de la declinación natural que ocurre con los tubérculos almacenados (Figura 1); Nemacur aplicado a UBV a las dosis de 12.5 y $25.0 \mathrm{cc} / \mathrm{l}$, no consiguió eliminar el nematodo de los tubérculos. Todos los productos químicos no mostraron efectos fitotóxicos en el brotamiento de tubérculos ni en el desarrollo de las plantas.

Se considera que el producto biológico Vertimec 018EC se presenta como una alternativa interesante para los productores de tubérculos-semillas por su baja toxicidad que no representa un peligro para la salud humana de los operarios y el medio ambiente. En general, en base a estos resultados debe tomarse en cuenta las consideraciones y/o recomendaciones que se indican a continuación para obtener resultados deseables:

El tratamiento a UBV de tubérculos infectados con $N$. aberrans con los productos Fostiazate 90EC y Vertimec 018EC, se debe limitar a aquellos tubérculos-semillas que procedan de campos que presenten una baja incidencia de plantas de papa infectadas ( $<10 \%$ de plantas con nodulación radical en relación al número total de plantas muestreadas por campo); y con una severidad incipiente (nodulación radical por planta $<5 \%$ de acuerdo al volumen total del sistema radical o de acuerdo al número de nódulos), que en este caso es menor a 10 nódulos por planta (Tabla 2), con el fin de evitar cualquier riesgo de escape. 
De ser deseable la ampliación de los límites de tolerancia indicados en el punto anterior, estos deberán ser establecidos por futuros estudios que se realicen con tubérculos que procedan de plantas con mayores niveles de incidencia y severidad de daño por $N$. aberrans.

Se recomienda además estudiar las dosis más bajas de Fostiazate 90EC y Vertimec 018EC, sin poner en riesgo su efectividad en la eliminación de nematodos en los tubérculos y ampliar el tratamiento de tubérculossemillas de papa a UBV de tubérculos infectados por $N$. aberrans de cultivares de la ssp. tuberosum, aun cuando ciertos antecedentes han mostrado una menor infección de este nematodo en tubérculos del cultivar Alpha (18).

\section{Agradecimiento}

Los autores agradecen a Daniel Blanc y Augusto Urquieta, funcionarios de la Unidad de Producción de Semilla de Papa-SEPA, por el apoyo brindado durante la ejecución del presente estudio.

\section{Referencias Bibliográficas}

1. Brodie,. B.B., K. Evans, J. Franco. 1993. Nematode parasites of potatoes. In: Plant parasitic nematodes in temperate agriculture. K.Evans, D. Trudgill and J. Webster (Eds.). CAB International, UK. p.87-117.

2. Costilla, M. 1985. El nematodo rosario o el falso nematodo del nódulo Nacobbus aberrans (Thorne, 1935) Thorne and Allen 1944, y su relación con el cultivo de papa en el noroeste argentino (Aspecto bioecológico, daño, dispersión y hospedantes). En: Investigaciones Nematológicas en Programas Latinoamericanos de Papa. J. Franco y H. Rincón (Eds.). CIP, Lima, Perú, pp.3-16.

3. Costilla, M., H. Basco. 1985. Experiencias de control químico del falso nematodo del nodulo Nacobbus aberrans (Thorne,1935) Thorne and Allen 1944, en tubérculos de papa en la Argentina. En: Investigaciones Nematológicas en Programas Latinoamericanos de Papa. J. Franco y H. Rincón (Eds.). CIP, Lima, Perú. p.17-20.

4. Franco, J. 1994. Problemas de nematodos en la producción de papa en climas templados en la región Andina. Nematrópica 24:179-195.

5. Franco, J., R. Oros, N. Ortuño. 1996. Consideraciones para el tratamiento químico de tubérculo semilla procedentes de campos infestados con Nacobbus aberrans. Memorias de la IV Reunión Nacional de la Papa, Cochabamba, Bolivia. p. 77-78. 
6. Franco, J., J. Ramos, R. Oros, G. Main, N. Ortuño. 1998/1999. Pérdidas económicas causadas por Nacobbus aberrans y Globodera spp. en el cultivo de la papa en Bolivia. Revista Latinoamericana de la Papa 11: 40-66.

7. Llano, M. A. 1999. Métodos para evaluar la eficiencia del tratamiento químico y físico de tubérculos-semillas de papa (Solanum tuberosum ssp. andigena) infectado con Nacobbus aberrans. Tesis Ing. Agr., Universidad Técnica de Oruro, Oruro, Bolivia. 79p.

8. Main, G., N. Ortuño, J. Franco. 2001. Protección de semilla de papa infectada con Nacobbus aberrans mediante nematicidas blandos. Manejo Integrado de Plagas (Costa Rica) 59:52-57.

9. Ministerio de Agricultura, Ganadería y Desarrollo Sostenible. 1999. Normas Específicas para la Certificación de Semilla de Papa. Resolución Ministerial N 101 del 1 de Octubre de 1999, La Paz, Bolivia. 11p.

10. Montalvo, R., J. Franco. 1994. Tratamiento de tubérculos-semilla con nematicidas. Informe Anual IBTA-PROINPA 1993-1994, Cochabamba, Bolivia. pp. IIIN-60.

11. Montecinos, R., J. Franco. 1993. .Diagnóstico de los principales nematodos del cultivo de la papa. Manual Técnico 1/93, IBTAPROINPA, Cochabamba, Bolivia. 26p.

12. Oros, R., G. Main, O. Diaz, J. Franco, N. Ortuño. 1996. Efecto de la aplicación de nematicidas en diferentes dosis en el cultivar W'aycha. Informe Anual IBTA-PROINPA 1995-1996, Cochabamba, Bolivia. p. IIIN-73.

13. Oros, R., M.A. Llano, F. Gallo. 1998. Tratamiento de tubérculos con productos naturales. Informe Anual IBTA-PROINPA 1997-1998, Cochabamba,Bolivia. p. IIIN-83.

14. Rojas, F., J. Franco, N. Ortuño. 1996/1997. Las ferias agrícolas: Fuente de diseminación de Nacobbus aberrans. Revista Latinoamericana de la Papa 9/10: 35-48.

15. Siles, E. 1999. Distribución horizontal de Nacobbus aberrans en parcelas de agricultores. Tesis Tec. Agr. Universidad de San Simón, Cochabamba, Bolivia. 62p.

16. Steel, R., J. Torrie. 1992. Bioestadística: Principios y procedimientos. 2da Ed. McGraw-Hill México. 620p.

17. Vargas, E., J. Franco, J. Blajos. 2002. Diagnóstico de los principales nematodos de la papa en las principales ferias agrícolas del departamento de La Paz, Bolivia (Por publicarse).

18. Villca, R., J. Franco, H. Equise. 2002. Evaluación de pérdidas causadas por Nacobbus aberrans en el cultivo de la papa. (Por publicarse). 